\title{
Push and Pull Factors Surrounding Older Adults' Relocation to Supportive Housing: A Scoping Review
}

\author{
Bryan B. Franco, ${ }^{1}$ Jason Randle, ${ }^{2}$ Lauren Crutchlow, ${ }^{3}$ Janet Heng, ${ }^{2}$ Arsalan Afzal, ${ }^{2}$ \\ George A. Heckman, ${ }^{4}$ and Veronique Boscart ${ }^{3,4}$ ()
}

\begin{abstract}
RÉSUMÉ
Les logements avec services de soutien, tels que les résidences pour personnes âgées et celles offrant des services d'assistance, sont de plus en plus considérés comme des choix adaptés aux besoins des personnes âgées au Canada. Cet examen de la portée décrit la nature et le contenu des recherches explorant les facteurs qui incitent les personnes âgées à déménager en résidences avec services de soutien. Une recherche effectuée sur PubMed, CINAHL, Web of Science et PsycINFO a permis de repérer 34 articles sur ce sujet. Les articles examinés utilisaient une variété de méthodes et de cadres théoriques, dont le modèle "Push and Pull", qui était le plus courant. Cet examen de la portée suggère que les problèmes de santé et les déficits fonctionnels sont les principales causes de relogement en résidences avec services. Davantage d'études longitudinales sont nécessaires en vue de définir de manière plus exhaustive les déterminants médicaux et sociaux du relogement et ses conséquences. Ceci permettra de caractériser plus précisément cette population en croissance pour mieux aligner les politiques sur les besoins des adultes âgés qui envisagent ou entreprennent un relogement.
\end{abstract}

\section{ABSTRACT}

Supportive housing, including retirement homes and assisted living, is increasingly touted as a suitable living option for Canadian older adults. This scoping review describes the nature and content of studies that explore underlying factors that motivate older adults to relocate to supportive housing. We conducted a search of PubMed, Cumulative Index of Nursing and Allied Health Literature (CINAHL), Web of Science, and PsycINFO, which identified 34 articles for review. Articles reviewed employed a variety of methods and guiding theoretical frameworks, of which the push and pull framework appeared to be most common. This review suggests that health and functional deficits are important reasons for relocation to supportive housing for older adults. Further longitudinal data are required to more comprehensively describe medical and social determinants for relocation and its consequences, in order to better describe this growing population and better align policies with the needs of older adults contemplating or undergoing relocation.

1 School of Medicine, Queen's University, Kingston, Ontario.

2 School of Public Health and Health Systems, University of Waterloo, Waterloo, Ontario.

3 Schlegel Centre for Advancing Seniors Care, Conestoga College, Kitchener, Ontario.

4 Schlegel-UW Research Institute for Aging, School of Public Health and Health Systems, University of Waterloo, Waterloo, Ontario.

* This work was supported by the Ministry of Health and Long-Term Care's 2017-2020 Health System Research Fund (HSRF; Grant \#255). G.A.H. receives salary support from the Schlegel-UW Research Institute for Aging. V.B. receives salary support from the Natural Science and Engineering Research Industrial Council of Canada (NSERC) through the Industrial Research Chair for Colleges (IRCC) Program, supported by Schlegel Villages and the Schlegel-UW Research Institute for Aging. The authors thank Dr. Heather Keller and Dr. Richard Hughson for their contributions.

Manuscript received: / manuscrit reçu : 16/07/2019

Manuscript accepted: / manuscrit accepté : 03/02/2020

Mots-clés: Vieillissement, personnes âgées, logement avec services de soutien, relogement, modèle push and pull, politique

Keywords: Aging, older adults, supportive housing, relocation, push and pull framework, policy

La correspondance et les demandes de tirés-à-part doivent être adressées à : / Correspondence and requests for offprints should be sent to:

Canadian Journal on Aging / La Revue canadienne du vieillissement 40 (2) : 263-281 (2021)

(C) Canadian Association on Gerontology 2020. This is an Open Access article, distributed under the terms of the Creative Commons Attribution licence (http://creativecommons.org/licenses/by/4.0/), which permits unrestricted re-use, distribution, and reproduction in any medium, provided the original work is properly cited. 
Veronique Boscart, Ph.D.

Schlegel-UW Research Institute for Aging

Schlegel Centre for Advancing Seniors Care

Conestoga College

299 Doon Valley Drive

Kitchener, Ontario, N2G 4M4

Canada

(vboscart@conestogac.on.ca)

\section{Introduction}

In 2016, more than 400,000 Canadians lived in longterm care or supportive housing (Statistics Canada, 2017a). As our population continues to age, an unprecedented number of individuals will come to live in such congregate arrangements (Statistics Canada, 2017b). Supportive housing is an umbrella term encompassing retirement homes and assisted living (Canadian Centre for Elder Law, 2008). Although some adults 65 years of age or older move to supportive housing from community living, supportive housing remains distinct from long-term care homes (Canadian Centre for Elder Law, 2008; Perks \& Haan, 2010). Long-term care homes are provincially regulated institutions that have entry requirements based on care needs, whereas supportive housing provides the option of relocating regardless of need (Howe, Jones, \& Tilse, 2013).

Within Canadian long-term care homes, person-level data are collected through the mandated use of a standardized instrument (the Resident Assessment Instrument/Minimum Data Set [RAI-MDS] 2.0 or interRAI-Long-Term Care Facilities [LTCF]) across provinces and territories (Hirdes, Mitchell, Maxwell, \& White, 2011). These data have played and continue to play a crucial role in shaping long-term care policy in Canada and other countries (Carpenter \& Hirdes, 2013). The lack of a similar data infrastructure in supportive housing impedes evidence-based policy discussions for a sector with fragmented and jurisdiction-specific regulations (Canadian Centre for Elder Law, 2008).

An aging population with increasing care needs places a growing emphasis on supportive housing as an alternative to long-term care (Perks \& Haan, 2010) but it is unknown whether these facilities have the capacity to meet residents' needs (Hirdes et al., 2011). For example, to be licensed in Ontario, a retirement home must offer at least two of the following services: meal provision, bathing assistance, personal hygiene, dressing or ambulation, dementia care, medication administration, incontinence care, or the services of a physician, nurse, or pharmacist (Ontario Retirement Communities Association, 2018). Thus, retirement homes may offer a wide range of heterogeneous services, such as providing meals and medication administration, but whether they can support residents who require additional help with activities of daily living is unclear. Without information on important factors that may be driving residents to relocate to supportive housing, it is difficult to ascertain the level of care needed to best serve this group of older adults.

A recent study from the Hamilton Niagara Haldimand Brant region of Ontario shed some light on the characteristics of retirement home residents by comparing those receiving home care services with home care clients living in the community (Poss et al., 2017). Approximately 40 per cent of retirement home residents receive home care services, and they tend to have greater cognitive and physical impairments than their community counterparts (Poss et al., 2017). This study also suggests that potential discrepancies exist between the care available in supportive housing and the needs of residents accessing such services. Yet, despite the growing numbers of older adults relocating to supportive housing, their characteristics and needs remain under-studied, hampering any informed assessment of the patchwork of policies implemented across Canada.

An understanding of the existing literature is needed to guide future investigations of prospective supportive housing residents, services, and policies. The push and pull framework, based on Lee's theory of migration (Lee, 1966), is a conceptual guide that is commonly used to examine the factors for relocation. In the context of older adults' relocation to supportive housing, this framework posits that older adults are influenced by push and pull factors when considering relocation to supportive housing. Pull factors are those that attract older adults to supportive housing, whereas push factors drive them out of their current living situation (Tyvimaa \& Kemp, 2011). Given the paucity of literature focused on this population, it is unclear how these factors relate to older adults' health and functioning; hindering the assessment of supportive housing policies' relative appropriateness.

We therefore conducted a scoping review to describe the nature and content of studies that explore older adults' reasons for relocating to supportive housing in order to better understand their needs. More specifically, we reviewed studies that examined older adults' reasons for moving, and their relation to health and function. 


\section{Methods}

A scoping review is designed to provide an overview of the literature on a topic with an expected paucity of evidence (Armstrong, Hall, Doyle, \& Waters, 2011). We conducted a scoping review of the supportive housing literature in accordance with Arksey and O'Malley's (2005) framework, to achieve our objectives.

\section{Search Strategy}

The search strategy was devised to describe the population, the setting, and the outcomes of interest. Because of the heterogeneity of terms used to describe supportive housing, a wide-range of keywords identified in Howe et al.'s (2013) international comparative search of terms was used (Table 1). We searched in PubMed, Cumulative Index of Nursing and Allied Health Literature (CINAHL), Web of Science, and PsycINFO up to and including August 2018.

\section{Article Selection}

We included journal articles that focused on individuals who were considering relocating to, or who were are already living in, supportive housing that were published in English between 2000 and August 2018, and for which a full-text version was available. Articles that studied naturally occurring retirement communities communities of older adults aging in a specific neighbourhood - were excluded because these are distinct from purpose-built supportive housing. Included articles were indexed and duplicates were removed using EndNote X7. Three authors independently screened articles' titles and abstracts and consulted a senior author to arbitrate screening decisions after full-text review.

\section{Data Charting and Thematic Analysis}

Publication characteristics, study characteristics, and participant information were collected and extracted. Publication characteristics included year of publication, journal, country in which the study was conducted, and MEDLINE ${ }^{\circledR}$ indexing status of the journal. Study characteristics included descriptors for supportive housing, study design, and the use of guiding frameworks or models, and participants' information included age, gender, and measures of health or functioning. MEDLINE indexing status was used as a surrogate for the visibility of the article to health care professionals and policy makers (Matsoukas, 2015).

Results were collated by identifying common themes in the literature (Levac, Colquhoun, \& O'Brien, 2010). Publication characteristics, general approaches in study designs, and the use of guiding frameworks or models were summarised once charted. Lastly, study

Table 1: Search strategy

\begin{tabular}{|c|c|c|}
\hline Concept & Search Terms & \\
\hline $\begin{array}{l}\text { Retirement home } \\
\text { AND Supportive } \\
\text { housing/Seniors }\end{array}$ & $\begin{array}{l}\text { (retirement AND (community OR communities OR home OR } \\
\text { village OR resort OR town OR condominium OR park OR } \\
\text { housing OR apartment)) OR } \\
\text { (village AND (lifestyle OR rental OR vertical)) OR } \\
\text { ((supportive OR supported) AND (housing OR senior OR } \\
\text { accommodation OR resident)) OR } \\
\text { (assisted AND (living OR service OR facility* OR apartment)) } \\
\text { OR } \\
\text { (residential AND (home OR care OR living OR park)) OR } \\
\text { (sheltered AND (care OR housing)) OR } \\
\text { (service AND (housing OR flat)) OR } \\
\text { (care AND (close OR flex OR integrated)) OR } \\
\text { ((seniors OR senior) AND housing) }\end{array}$ & $\begin{array}{l}\text { TS= ((retirement AND (community OR communities OR home OR } \\
\text { village OR resort OR town OR condominium OR park OR } \\
\text { housing OR apartment)) OR (village AND (lifestyle OR rental OR } \\
\text { vertical)) OR ((supportive OR supported) AND (housing OR } \\
\text { senior OR accommodation OR resident)) OR ((assisted AND } \\
\text { (living OR service OR facility* OR apartment)) OR (residential } \\
\text { AND (home OR care OR living OR park)) OR (sheltered AND } \\
\text { (care OR housing)) OR (service AND (housing OR flat)) OR (care } \\
\text { AND (close OR flex OR integrated)) OR ((seniors OR senior) AND } \\
\text { housing))) }\end{array}$ \\
\hline Push and pull factors & Retirement home AND & $\begin{array}{l}\text { (relocation OR mobility OR move OR moving OR migrate OR } \\
\text { migration OR transition) AND } \\
\text { (choice OR consider* OR decision OR decid* OR select* OR } \\
\text { reason* OR push OR pull OR proactive OR reactive OR plan OR } \\
\text { influence OR determin* OR factor) }\end{array}$ \\
\hline $\begin{array}{l}\text { Disease, functioning, } \\
\text { and frailty }\end{array}$ & Retirement home AND & $\begin{array}{l}\text { (disease OR illness OR comorbidity OR frailty OR Function* OR } \\
\text { Activities of daily living OR ADL* OR IADL* OR Instrumental } \\
\text { activities of daily living OR Quality of life OR Functional status } \\
\text { OR everyday function* OR Functionally impaired elder*) AND } \\
\text { (cohort OR cross-section OR survey OR review) AND } \\
\text { (prevalence OR incidence OR epidemiology OR risk) }\end{array}$ \\
\hline
\end{tabular}


participants were described, and measures of health and function were reported.

\section{Results}

\section{Search Results}

Our database searches returned 15,522 publications, with 13,615 unique citations. A total of 2677 (not counting 128 duplicates) were added after the updated search in August 2018. After screening and full-text review, 34 articles were included (Figure 1).

\section{Summary of Study Characteristics}

Table 2 provides an overview of included articles' study characteristics. Most studies (44\%) were conducted it the United States, followed by Australia (21\%), Canada $(12 \%)$, and Europe (12\%). Two studies (6\%) were conducted each in Israel, China, and Taiwan. More than half of articles (56\%) were published between 2007 and 2012, with only 12 per cent having been published before 2006. The majority of studies (65\%) were published in journals that were indexed for MEDLINE. Articles were most frequently published in the Journal of Applied Gerontology (15\%) and Journal of Housing for the Elderly (12\%). The former is indexed for MEDLINE but the latter is not.

The most frequently used descriptor for supportive housing was retirement living, with 44 per cent of studies using "retirement" in their definition of their setting, followed by "assisted living" (21\%). Settings that included "retirement" were continuing care retirement communities, retirement villages, and retirement communities. Remaining articles (35\%) used a variety of descriptors, such as senior housing/houses (including congregated senior housing and housing for seniors), supportive housing, and government-subsidized senior citizen apartment buildings (Table 2).

The push and pull framework was the most commonly applied framework (27\%); half of studies used another conceptual approach, and 24 per cent used none. Almost all 34 articles reported participants' age and gender, and 65 per cent reported at least one measure of health and/or functioning.

\section{Study Designs}

Studies used various designs to explore older adults' factors for relocating to supportive housing. A qualitative approach was applied in half of the studies, while quantitative approaches were used in 47 per cent (Table 2). Only one study used mixed methods, in which they conducted interviews and applied quantitative instruments (Ewen \& Chahal, 2013).

Qualitative approaches consisted of interviews with older adults and/or their families who were planning to move to, or already resided in, supportive housing. Most authors analyzed qualitative data using thematic or content analysis (Table 3). A few studies also collected data via participant observation. These studies

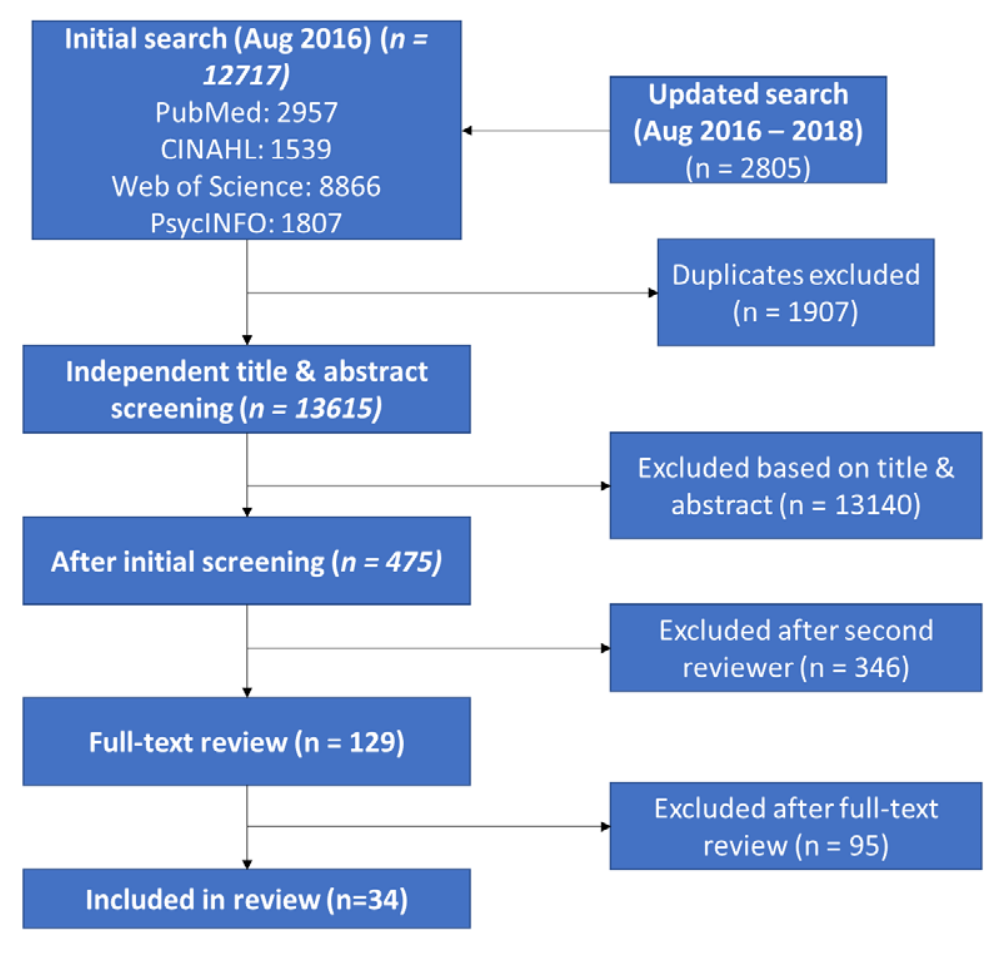

Figure 1: Overview of Article Selection Process 
Table 2: Study characteristics

\begin{tabular}{|c|c|}
\hline & Number of Studies (\%) \\
\hline \multicolumn{2}{|l|}{ Year of publication } \\
\hline 2000-2006 & $4(12 \%)$ \\
\hline $2007-2012$ & $19(56 \%)$ \\
\hline $2013-2018$ & $11(32 \%)$ \\
\hline \multicolumn{2}{|l|}{ Country } \\
\hline United States & $15(44 \%)$ \\
\hline Australia & $7(21 \%)$ \\
\hline Europe & $4(12 \%)$ \\
\hline Canada & $4(12 \%)$ \\
\hline Other & $4(12 \%)$ \\
\hline Indexed for MEDLINE ${ }^{\circledR}$ & $22(65 \%)$ \\
\hline \multicolumn{2}{|l|}{ Supportive housing descriptors } \\
\hline Retirement living & $15(44 \%)$ \\
\hline Assisted living & $7(21 \%)$ \\
\hline Other descriptors & $12(35 \%)$ \\
\hline \multicolumn{2}{|l|}{ Study design } \\
\hline Qualitative & $17(50 \%)$ \\
\hline Quantitative & $16(47 \%)$ \\
\hline Mixed methods & $1(3 \%)$ \\
\hline \multicolumn{2}{|l|}{ Guiding frameworks and models } \\
\hline Push and pull framework & $9(27 \%)$ \\
\hline Ecological theory of aging & $6(18 \%)$ \\
\hline Other & $11(32 \%)$ \\
\hline None & $8(24 \%)$ \\
\hline Studies reporting age of participants & $33(97 \%)$ \\
\hline Studies reporting gender of participants & $34(100 \%)$ \\
\hline Studies with measures of health or functioning & $22(65 \%)$ \\
\hline General health & $21(62 \%)$ \\
\hline ADLs and/or IADLs & $11(32 \%)$ \\
\hline Depression or mood & $6(18 \%)$ \\
\hline Cognition & $4(12 \%)$ \\
\hline Pain & $3(9 \%)$ \\
\hline No assessment of health or functioning & $12(35 \%)$ \\
\hline
\end{tabular}

Note. $\mathrm{ADL}=$ activities of daily living; $\mathrm{IADL}=$ instrumental activities of daily living.

were guided by a phenomenological approach (Jungers, 2010), a grounded theory approach (Ball, Perkins, Hollingsworth, Whittington, \& King, 2009), and a thematic analysis through micro, meso, and macro lenses (Portacolone \& Halpern, 2016).

Quantitative approaches largely entailed the use of surveys and questionnaires developed by authors for the purposes of the study (Table 3). The majority of studies used a cross-sectional design to look at relationships between factors surrounding the transition, but a few studies were longitudinal in nature. A pair of studies, for example, administered a survey at two time points, one year apart, to investigate older adults' relocation outcomes after moving to supportive housing (Smith \& Sylvestre, 2008; Sylvestre \& Smith, 2009). Two studies used longitudinal data from existing cohorts: the Longitudinal Study on Aging II in the United States (Hong \& Chen, 2009) and the ENABLEAGE Project in Europe (Granbom et al., 2014). Finally, one study used online vignettes to present different scenarios to prospective older adults and their adult children to explore decision-making surrounding relocation (Caro et al., 2012).

\section{Guiding Theoretical Frameworks}

The majority of studies (77\%) used a theoretical framework to guide their inquiry into older adults' relocation to supportive housing (Table 2). The most frequently used framework was the push and pull framework, which was applied explicitly in 27 per cent of studies, followed by the ecological theory of ageing, which was used in 18 per cent of studies. Different frameworks were used in 32 per cent of articles. The rest of the articles did not report the use of a framework. Table 4 lists these theoretical frameworks.

Most frameworks applied in the studies reviewed were directly related to ageing and relocation: the push and pull framework, the ecological theory of ageing, or frameworks describing different types of movers (e.g. Litwak and Longino Jr's (1987) and Gardner, Browning, and Kendig's (2005) models, and concepts designed for examining person-environment interactions (e.g. complementary/congruence model of wellbeing). However, some researchers generalised concepts that are non-specific to older adults or relocation to study this phenomenon, including Rosenbaum's (1990) theory of learned resourcefulness, grief, ecological system theory (Portacolone \& Halpern, 2016), and the theory of planned behaviour (Ajzen, 1985).

\section{Push and Pull Factors Affecting Relocation to Supportive Housing}

The articles that applied the push and pull framework revealed several factors involved in older adults' relocation to supportive housing (Table 2). Push factors for relocation included individuals' or spouses' health challenges, increasing social isolation, fear of burdening family, inadequate living arrangements, necessary maintenance of property, and aiming to achieve control over one's future. The most frequently cited push factor in the studies were older adult's or their spouse's declining health (Bekhet, Zauszniewski, \& Nakhla, 2009; Crisp, Windsor, Anstey, \& Butterworth, 2013; Ewen \& Chahal, 2013; Groger \& Kinney, 2001; Stimson \& McCrea, 2004; Tyvimaa \& Kemp, 2011). Articles that did not explicitly use the push and pull framework also showed that older adults who were relocated experienced increasing physical decline (Svidén, Wikström, \& Hjortsjö-Norberg, 2002), falls (Castle \& Sonon, 2007; Saunders \& Heliker, 2008), cognitive impairment (Rockwood et al., 2014), and/or functional deficits (Ewen \& Chahal, 2013; Granbom, Lofqvist, Horstmann, Haak, \& Iwarsson, 2014). 
Table 3: Summary table of reviewed studies

\begin{tabular}{|c|c|c|c|c|c|c|c|}
\hline Authors (Year) & $\begin{array}{l}\text { Descriptor for Sup- } \\
\text { portive Housing }\end{array}$ & Purpose of Study & $\begin{array}{l}\text { Guiding Frame- } \\
\text { works or Models }\end{array}$ & $\begin{array}{l}\text { Data Collection Methods } \\
\text { and Tools }\end{array}$ & Data Analysis & $\begin{array}{l}\text { Participants or } \\
\text { Sample }\end{array}$ & $\begin{array}{l}\text { Age Reported } \\
\text { (Years) }\end{array}$ \\
\hline $\begin{array}{l}\text { Groger and Kin- } \\
\text { ney (2001) }\end{array}$ & $\begin{array}{l}\text { Continuing care } \\
\text { retirement commu- } \\
\text { nity (CCRC) }\end{array}$ & $\begin{array}{l}\text { To better understand why } \\
\text { people move to CCRCs }\end{array}$ & Push-pull framework & $\begin{array}{l}\text { Interviews } \\
\text { Functional health: (Short } \\
\text { Form Survey-36 [SF-36]); } \\
\text { cognitive functioning } \\
\text { (Metamemory Question- } \\
\text { naire); depression (The } \\
\text { Center for Epidemiologic } \\
\text { Studies Depression Scale } \\
\text { [CES-D]) }\end{array}$ & Thematic analysis & $\begin{array}{l}8 \text { married couples and } \\
5 \text { single women } \\
\text { moving into CCRCs }\end{array}$ & $\begin{array}{l}\text { Mean: } 74.4 \text { years } \\
\text { (Range: } 68-81 \\
\quad \text { years) }\end{array}$ \\
\hline $\begin{array}{l}\text { Svidén, Wikström, } \\
\text { and Hiortsj- } \\
\text { ö-Norberg } \\
\text { (2002) }\end{array}$ & $\begin{array}{l}\text { Sheltered housing for } \\
\text { the elderly }\end{array}$ & $\begin{array}{l}\text { To analyse how individ- } \\
\text { uals describe their } \\
\text { experience of moving } \\
\text { to and living in shel- } \\
\text { tered housing }\end{array}$ & $\mathrm{N} / \mathrm{A}$ & Semi-structured interviews & $\begin{array}{l}\text { Phenomenological } \\
\text { approach to } \\
\text { thematic ana- } \\
\text { lysis }\end{array}$ & $\begin{array}{l}41 \text { women and } 18 \\
\text { men living in shel- } \\
\text { tered housing for } \\
>1 \text { year }\end{array}$ & Not reported \\
\hline Krout et al. (2002) & CCRC & $\begin{array}{l}\text { To examine reasons given } \\
\text { for relocation to an } \\
\text { upstate New York } \\
\text { CCRC }\end{array}$ & $\begin{array}{l}\text { Push-pull framework } \\
\text { Litwak and Longino's } \\
\text { model } 3 \text { types of } \\
\text { moves among the } \\
\text { elderly }\end{array}$ & $\begin{array}{l}\text { Interviews and author- } \\
\text { developed survey to } \\
\text { collect demographics } \\
\text { Self-assessed health on a } \\
10 \text {-point scale ( } 0=\text { very } \\
\text { serious health problems } \\
\text { to } 10=\text { very best health); } \\
\text { spouse's assessed health } \\
\text { using the same } 10 \text {-point } \\
\text { scale }\end{array}$ & $\begin{array}{l}\text { Exploratory factor } \\
\text { analyses and } \\
\text { multiple logistic } \\
\text { regressions }\end{array}$ & $\begin{array}{l}91 \text { affluent individuals } \\
\text { who relocated to } \\
\text { one CCRC (64\% } \\
\text { female and had } \\
\text { graduate/profes- } \\
\text { sional degrees) }\end{array}$ & $60 \%>75$ years \\
\hline $\begin{array}{l}\text { Stimson and } \\
\text { McCrea (2004) }\end{array}$ & Retirement village & $\begin{array}{l}\text { To identify relationships } \\
\text { between push-pull fac- } \\
\text { tors, predictor vari- } \\
\text { ables, and relocation to } \\
\text { retirement villages }\end{array}$ & Push-pull framework & Mail-in survey & $\begin{array}{l}\text { Factor analyses } \\
\text { and path ana- } \\
\text { lyses }\end{array}$ & $\begin{array}{l}985 \text { residents typically } \\
\text { from a white-collar } \\
\text { background } \\
\text { (60-65\% female) }\end{array}$ & $\begin{array}{l}\text { Most common age } \\
\text { of entry is } 70- \\
74 \text { years }\end{array}$ \\
\hline $\begin{array}{l}\text { Bekhet, Zausz- } \\
\text { niewski, and } \\
\text { Wykle (2008) }\end{array}$ & $\begin{array}{l}\text { Retirement communi- } \\
\text { ties }\end{array}$ & $\begin{array}{l}\text { To examine the relation- } \\
\text { ship among positive } \\
\text { cognitions, learned } \\
\text { resourcefulness, and } \\
\text { relocation adjustments }\end{array}$ & $\begin{array}{l}\text { Rosenbaum's theory } \\
\text { of learned } \\
\text { resourcefulness }\end{array}$ & $\begin{array}{l}\text { Self-report questionnaire } \\
\text { with scales to assess } \\
\text { learned resourcefulness, } \\
\text { positive cognition, and } \\
\text { relocation adjustment } \\
\text { Perceived Health Status } \\
\text { (4-point Likert scale, } \\
1=\text { poor } 4=\text { excellent); } \\
\text { Index of Activities of } \\
\text { Daily living (lower scor- } \\
\text { e=more independence) }\end{array}$ & $\begin{array}{l}\text { Hierarchal regres- } \\
\text { sion and a cor- } \\
\text { relation matrix }\end{array}$ & $\begin{array}{l}104 \text { cognitively unim- } \\
\text { paired elders (66\% } \\
\text { women) }\end{array}$ & $\begin{array}{l}\text { Mean: } 85 \text { years } \\
\text { (range: } 65-95 \\
\text { years) }\end{array}$ \\
\hline
\end{tabular}


Table 3: Continued

\begin{tabular}{|c|c|c|c|c|c|c|c|}
\hline Authors (Year) & $\begin{array}{l}\text { Descriptor for Sup- } \\
\text { portive Housing }\end{array}$ & Purpose of Study & $\begin{array}{l}\text { Guiding Frame- } \\
\text { works or Models }\end{array}$ & $\begin{array}{l}\text { Data Collection Methods } \\
\text { and Tools }\end{array}$ & Data Analysis & $\begin{array}{l}\text { Participants or } \\
\text { Sample }\end{array}$ & $\begin{array}{l}\text { Age Reported } \\
\text { (Years) }\end{array}$ \\
\hline $\begin{array}{l}\text { Bekhet, Zausz- } \\
\text { niewski, and } \\
\text { Nakhla (2009) }\end{array}$ & & $\begin{array}{l}\text { To understand why elders } \\
\text { relocate to retirement } \\
\text { communities and what } \\
\text { living in the communi- } \\
\text { ties is like from their } \\
\text { perspective }\end{array}$ & Push-pull framework & Interviews & & $\begin{array}{l}\text { Constant compara- } \\
\text { tive method }\end{array}$ & \\
\hline $\begin{array}{l}\text { Castle and Sonon } \\
\text { (2007) }\end{array}$ & Assisted living facilities & $\begin{array}{l}\text { To examine factors } \\
\text { around the search, } \\
\text { selection, and satisfac- } \\
\text { tion of residents and } \\
\text { family members in } \\
\text { assisted living }\end{array}$ & $\begin{array}{l}\text { Conceptual model } \\
\text { based on typical } \\
\text { sequence for } \\
\text { assisted living } \\
\text { search and selec- } \\
\text { tion process }\end{array}$ & $\begin{array}{l}\text { Questionnaire adopted } \\
\text { from nursing home } \\
\text { research, consisting of } \\
\text { health and functional } \\
\text { measures, demograph- } \\
\text { ics, search, choice, selec- } \\
\text { tion, and satisfaction } \\
\text { Self-reported health status } \\
\text { (SF-36, } 4-20 \text { with higher } \\
\text { scores meaning better } \\
\text { health); ADL status } \\
\text { (higher scores = less } \\
\text { dependence); IADL sta- } \\
\text { tus (higher scores=less } \\
\text { dependence); mood } \\
\text { (0-30 mood scale; higher } \\
=\text { better mood); pain (1 } \\
=\text { no pain, } 6=\text { unbear- } \\
\text { able pain }\end{array}$ & Logistic regression & $\begin{array}{l}375 \text { assisted living } \\
\text { residents (76\% } \\
\text { female) with } \\
\text { matched family } \\
\text { members ( } 76 \% \\
\text { female) }\end{array}$ & 77 years (SD: 5.2 ) \\
\hline Kemp (2008) & Assisted living facilities & $\begin{array}{l}\text { To explore pathways that } \\
\text { lead couples to assisted } \\
\text { living and how mar- } \\
\text { riage influences their } \\
\text { lives in this setting }\end{array}$ & N/A & Interviews & Inductive analysis & $\begin{array}{l}20 \text { married couples } \\
\text { and } 10 \text { adult chil- } \\
\text { dren }\end{array}$ & $\begin{array}{l}\text { Husband: mean } \\
86 \text { years (range } \\
66-94 \text { ) } \\
\text { Wife age: mean } \\
85 \text { years (range } \\
67-93 \text { ) }\end{array}$ \\
\hline $\begin{array}{l}\text { Saunders and } \\
\text { Heliker (2008) }\end{array}$ & Assisted living facility & $\begin{array}{l}\text { To explore the expect- } \\
\text { ations and experiences } \\
\text { of newly admitted resi- } \\
\text { dents }\end{array}$ & $\mathrm{N} / \mathrm{A}$ & Interviews & Content analysis & $\begin{array}{l}5 \text { newly admitted } \\
\text { female residents }\end{array}$ & $\begin{array}{c}\text { Mean: } 78.9 \text { years } \\
\text { (range 63-91) }\end{array}$ \\
\hline $\begin{array}{l}\text { Sergeant and } \\
\quad \text { Ekerdt (2008) }\end{array}$ & $\begin{array}{l}\text { Congregated senior } \\
\text { housing, assisted } \\
\text { living }\end{array}$ & $\begin{array}{l}\text { To study motives for resi- } \\
\text { dential mobility and the } \\
\text { decision-making pro- } \\
\text { cess within the context } \\
\text { of the ecological layers } \\
\text { of aging }\end{array}$ & $\begin{array}{l}\text { Ecological layers of } \\
\text { the aging context }\end{array}$ & $\begin{array}{l}\text { Interviews } \\
\text { Self-reported health prob- } \\
\text { lems }\end{array}$ & Thematic analysis & $\begin{array}{l}30 \text { individuals and } \\
\text { couples who } \\
\text { moved within the } \\
\text { past year and } 14 \\
\text { extended family } \\
\text { members ( } 66 \% \\
\text { female). }\end{array}$ & $\begin{array}{l}11 \% 60-69 \text { years } \\
39 \% 70-79 \text { years } \\
50 \% 80-87 \text { years }\end{array}$ \\
\hline
\end{tabular}




\begin{tabular}{|c|c|c|c|c|c|c|c|}
\hline Authors (Year) & $\begin{array}{l}\text { Descriptor for Sup- } \\
\text { portive Housing }\end{array}$ & Purpose of Study & $\begin{array}{l}\text { Guiding Frame- } \\
\text { works or Models }\end{array}$ & $\begin{array}{l}\text { Data Collection Methods } \\
\text { and Tools }\end{array}$ & Data Analysis & $\begin{array}{l}\text { Participants or } \\
\text { Sample }\end{array}$ & $\begin{array}{l}\text { Age Reported } \\
\text { (Years) }\end{array}$ \\
\hline $\begin{array}{l}\text { Smith and Sylves- } \\
\text { tre (2008) }\end{array}$ & $\begin{array}{l}\text { Government subsid- } \\
\text { ized senior citizen } \\
\text { apartment build- } \\
\text { ings }\end{array}$ & $\begin{array}{l}\text { To determine effects of } \\
\text { neighbourhood and } \\
\text { individual change on } \\
\text { personal outcomes of } \\
\text { recent movers }\end{array}$ & $\begin{array}{l}\text { Amended Golant's } \\
\text { model, an inter- } \\
\text { actional world- } \\
\text { view in which older } \\
\text { adults' relocation } \\
\text { outcomes are } \\
\text { influenced by per- } \\
\text { sonal and } \\
\text { behaviour-setting } \\
\text { components }\end{array}$ & $\begin{array}{l}\text { Investigator-developed } \\
\text { longitudinal survey } \\
\text { administered } 1 \text { year } \\
\text { apart } \\
\text { Self-rated health (5-point } \\
\text { scale from bad to excel- } \\
\text { lent); depression (CES-D); } \\
\text { Index of ADLs (11 ADLs, } \\
\text { Katz et al.); IADL Scale (8 } \\
\text { tasks, Lawton and Brody) }\end{array}$ & Logistic regression & $\begin{array}{l}137 \text { retirees (62\% } \\
\text { women) who } \\
\text { recently (1 month to } \\
1 \text { year) moved to } \\
\text { senior citizen } \\
\text { apartment build- } \\
\text { ings. Majority of } \\
\text { respondents } \\
\text { obtained a gr } 7 \text { to } \\
12 \text { education. }\end{array}$ & $\begin{array}{l}37 \% 55-64 \text { years } \\
27 \% 65-74 \text { years } \\
25 \% 75-84 \text { years } \\
11 \% \geq 85 \text { years }\end{array}$ \\
\hline $\begin{array}{l}\text { Sylvestre and } \\
\text { Smith (2009) }\end{array}$ & & $\begin{array}{l}\text { To determine effects of } \\
\text { changes in local } \\
\text { behaviour settings and } \\
\text { individual attributes on } \\
\text { adjustments of older } \\
\text { parents who have } \\
\text { moved to senior citizen } \\
\text { apartment buildings }\end{array}$ & & & & & \\
\hline Ball et al. (2009) & Assisted living facilities & $\begin{array}{l}\text { To examine how race and } \\
\text { class influence decisions } \\
\text { to move to assisted liv- } \\
\text { ing }\end{array}$ & N/A & $\begin{array}{l}\text { Interviews, participant } \\
\text { observation, and review } \\
\text { of residents' records } \\
\text { Self-reported health prob- } \\
\text { lems }\end{array}$ & $\begin{array}{l}\text { Grounded theory } \\
\text { approach }\end{array}$ & $\begin{array}{l}60 \text { residents }(70 \% \\
\text { women), } 43 \text { family } \\
\text { members/friends, } \\
12 \text { administrators; } \\
\sim 50 \% \text { African } \\
\text { American and } 50 \% \\
\text { white }\end{array}$ & $\begin{array}{l}4 \% 18-44 \text { years } \\
11 \% 45-64 \text { years } \\
10 \% 65-74 \text { years } \\
46 \% 75-84 \text { years } \\
29 \% \geq 85 \text { years }\end{array}$ \\
\hline $\begin{array}{l}\text { Hong and Chen } \\
\text { (2009) }\end{array}$ & Supportive housing & $\begin{array}{l}\text { To test a model for late- } \\
\text { life relocation and } \\
\text { health derived from the } \\
\text { ecological model of } \\
\text { aging and the comple- } \\
\text { mentary/ congruence } \\
\text { model of well-being }\end{array}$ & $\begin{array}{l}\text { Ecological model of } \\
\text { aging } \\
\text { Complementary/ } \\
\text { congruence model } \\
\text { of well-being }\end{array}$ & $\begin{array}{l}\text { Longitudinal Study on } \\
\text { Aging II (a nationally } \\
\text { representative sample of } \\
\text { noninstitutionalized per- } \\
\text { sons } \geq 70 \text { years) } \\
\text { Looked at } 12 \text { medical con- } \\
\text { ditions for comorbidities; } \\
\text { functional disability } \\
\text { (Nagi's } 7 \text { items); Func- } \\
\text { tional limitation (com- } \\
\text { bined ADLs, IADLs); Self- } \\
\text { rated health ( } 1=\text { excellent } \\
\text { to } 5=\text { poor) }\end{array}$ & $\begin{array}{l}\text { Latent growth } \\
\text { curve modeling } \\
\text { (to estimate } \\
\text { variations in ini- } \\
\text { tial status and } \\
\text { longitudinal } \\
\text { changes) }\end{array}$ & $\begin{array}{l}5,294 \text { older adults } \\
\text { (63\% women); } \\
2.4 \% \text { recently } \\
\text { relocated to sup- } \\
\text { portive housing }\end{array}$ & $\begin{array}{l}\text { Mean: } 75.5 \text { years } \\
\text { (SD: } 5.26)\end{array}$ \\
\hline Jungers (2010) & Assisted living & $\begin{array}{l}\text { To create a rich descrip- } \\
\text { tion of the meaning of } \\
\text { relocation and describe } \\
\text { nuances in the process }\end{array}$ & Late-life transition & $\begin{array}{l}\text { Focus group, interviews, } \\
\text { and observations }\end{array}$ & $\begin{array}{l}\text { Phenomenological } \\
\text { approach to } \\
\text { thematic } \\
\text { analysis }\end{array}$ & $\begin{array}{l}14 \text { participants (10 } \\
\text { women) residing in } \\
\text { assisted living facil- } \\
\text { ities }\end{array}$ & $\begin{array}{c}\text { Mean: } 85.2 \text { years } \\
\text { (range: } 75-98 \text { ) }\end{array}$ \\
\hline
\end{tabular}


Table 3: Continued

\begin{tabular}{|c|c|c|c|c|c|c|c|}
\hline Authors (Year) & $\begin{array}{l}\text { Descriptor for Sup- } \\
\text { portive Housing }\end{array}$ & Purpose of Study & $\begin{array}{l}\text { Guiding Frame- } \\
\text { works or Models }\end{array}$ & $\begin{array}{l}\text { Data Collection Methods } \\
\text { and Tools }\end{array}$ & Data Analysis & $\begin{array}{l}\text { Participants or } \\
\text { Sample }\end{array}$ & $\begin{array}{l}\text { Age Reported } \\
\text { (Years) }\end{array}$ \\
\hline $\begin{array}{l}\text { Cheng et al. } \\
\text { (2011) }\end{array}$ & $\begin{array}{l}\text { Residential care facil- } \\
\text { ities }\end{array}$ & $\begin{array}{l}\text { To understand the well- } \\
\text { being of residents and } \\
\text { how the environment } \\
\text { affects residents' activ- } \\
\text { ities and health }\end{array}$ & $\begin{array}{l}\text { Therapeutic land- } \\
\text { scapes (Gesler) }\end{array}$ & $\begin{array}{l}\text { Interviews } \\
\text { Self-rated health status } \\
\text { (5-point scale from } \\
\text { excellent to poor) }\end{array}$ & $\begin{array}{l}\text { Constant com- } \\
\text { parative } \\
\text { method }\end{array}$ & $\begin{array}{l}27 \text { elderly residents } \\
\text { (17 female), } 16 \\
\text { family members, } 5 \\
\text { residential care } \\
\text { facility managers }\end{array}$ & $\begin{array}{l}\text { Mean age: } 80 \\
\text { years }\end{array}$ \\
\hline Finn et al. (2011) & Retirement village & $\begin{array}{l}\text { To explore financial con- } \\
\text { siderations that retirees } \\
\text { had when relocating }\end{array}$ & N/A & Convergent interviews & Content analysis & $\begin{array}{l}81 \text { retirement village } \\
\text { residents; } 52 \text { dis- } \\
\text { cussed financial } \\
\text { issues ( } 28 \text { women) } \\
\text { whose interviews } \\
\text { were analyzed }\end{array}$ & $\begin{array}{r}\text { Mean } 76.3 \text { years } \\
\text { (range: } 55-89 \text { ) }\end{array}$ \\
\hline $\begin{array}{l}\text { Tyvimaa and } \\
\text { Kemp (2011) }\end{array}$ & Senior house & $\begin{array}{l}\text { To explore factors influ- } \\
\text { encing relocation to } \\
\text { senior houses }\end{array}$ & Push-pull framework & $\begin{array}{l}\text { Interviews and open-ended } \\
\text { questions from surveys }\end{array}$ & Content analysis & $\begin{array}{l}120 \text { senior housing } \\
\text { residents (15 were } \\
\text { interviewed) from } 3 \\
\text { sites; } 55-79 \% \text { were } \\
\text { women depending } \\
\text { on site }\end{array}$ & $\begin{array}{l}\text { At site } 1,50 \% \text { of } \\
\text { respondents } \\
\text { were } \geq 75 \\
\text { years. } \\
\text { At site } 2 \text {, respond- } \\
\text { ents were } \\
60-70 \text { years } \\
\text { old. } \\
\text { At site } 3 \text {, age was } \\
\text { distributed from } \\
55 \text { to } \geq 75 \text { years }\end{array}$ \\
\hline $\begin{array}{l}\text { Ayalon and } \\
\text { Green (2012) }\end{array}$ & CCRC & $\begin{array}{l}\text { To examine the transition } \\
\text { to CCRCs within the } \\
\text { framework of antici- } \\
\text { patory and disenfran- } \\
\text { chised grief }\end{array}$ & $\begin{array}{l}\text { Anticipatory and dis- } \\
\text { enfranchised grief }\end{array}$ & Interviews & Thematic analysis & $\begin{array}{l}29 \text { CCRC residents ( } 24 \\
\text { female) and } 19 \\
\text { adult children (13 } \\
\text { female) }\end{array}$ & $\begin{array}{l}\text { Range: } 72-88 \\
\quad \text { years }\end{array}$ \\
\hline $\begin{array}{l}\text { Bäumker et al. } \\
\text { (2012) }\end{array}$ & Extra care housing & $\begin{array}{l}\text { To examine factors } \\
\text { motivating older } \\
\text { people to move to } \\
\text { extra care housing } \\
\text { compared with those } \\
\text { moving to retirement } \\
\text { villages }\end{array}$ & Push-pull framework & $\begin{array}{l}\text { Investigator-developed } \\
\text { questionnaire } \\
\text { Self-perceived health } \\
\text { (5-point scale, very bad } \\
\text { to very good); ADLs } \\
\text { (Barthel Index of ADLs); } \\
\text { cognitive impairment } \\
\text { (Minimum Data Set } \\
\text { Cognitive Performance } \\
\text { Scale [MDS-CPS]) }\end{array}$ & $\begin{array}{l}\text { Chi-square ana- } \\
\text { lyses }\end{array}$ & $\begin{array}{l}949 \text { individuals (65\% } \\
\text { female) recently } \\
\text { relocated to extra } \\
\text { care housing or } \\
\text { retirement villages }\end{array}$ & $\begin{array}{l}\text { Mean ages } \\
\text { among settings } \\
\text { ranged from } \\
75.5 \text { to } 77.5 \\
\text { years }\end{array}$ \\
\hline
\end{tabular}


Table 3: Continued

\begin{tabular}{|c|c|c|c|c|c|c|c|}
\hline Authors (Year) & $\begin{array}{l}\text { Descriptor for Sup- } \\
\text { portive Housing }\end{array}$ & Purpose of Study & $\begin{array}{l}\text { Guiding Frame- } \\
\text { works or Models }\end{array}$ & $\begin{array}{l}\text { Data Collection Methods } \\
\text { and Tools }\end{array}$ & Data Analysis & $\begin{array}{l}\text { Participants or } \\
\text { Sample }\end{array}$ & $\begin{array}{l}\text { Age Reported } \\
\text { (Years) }\end{array}$ \\
\hline Caro et al. (2012) & Retirement community & $\begin{array}{l}\text { To determine how five } \\
\text { distinct dimensions } \\
\text { (functional status, fea- } \\
\text { tures of current hous- } \\
\text { ing, social networks, } \\
\text { features of retirement } \\
\text { communities, and } \\
\text { finances) affect deci- } \\
\text { sions to relocate }\end{array}$ & $\begin{array}{l}\text { Ecological theory of } \\
\text { aging }\end{array}$ & $\begin{array}{l}\text { Online vignettes (text, } \\
\text { photographs, audiovi- } \\
\text { sual clips) using a frac- } \\
\text { tional factorial survey } \\
\text { design } \\
\text { Self-reported health status; } \\
\text { IADLs }\end{array}$ & Logistic regression & $\begin{array}{l}215 \text { older adults }(79 \% \\
\text { female) and } 51 \\
\text { adult children (6\% } \\
\text { in senior housing) }\end{array}$ & Median: 73 years \\
\hline Huang (2012) & Senior housing & $\begin{array}{l}\text { To use a model based on } \\
\text { the theory of planned } \\
\text { behaviour to investi- } \\
\text { gate factors underlying } \\
\text { older adults' intention } \\
\text { to move into senior } \\
\text { housing }\end{array}$ & $\begin{array}{c}\text { Theory of planned } \\
\text { behaviour }\end{array}$ & $\begin{array}{l}\text { Questionnaire based on the } \\
\text { model developed using } \\
\text { the theory of planned } \\
\text { behaviour } \\
\text { Self-reported health status }\end{array}$ & $\begin{array}{l}\text { Predictive model- } \\
\text { ing }\end{array}$ & $\begin{array}{l}264 \text { older adults (63\% } \\
\text { female) from } 5 \\
\text { randomly selected } \\
\text { in older adult } \\
\text { schools }\end{array}$ & $\begin{array}{l}28 \% 61-65 \text { years } \\
21 \% 66-70 \text { years } \\
20 \% 71-75 \text { years } \\
20 \% 76-80 \text { years } \\
11 \% \geq 81 \text { years }\end{array}$ \\
\hline $\begin{array}{l}\text { Weeks, Keefe, } \\
\text { and Macdo- } \\
\text { nald (2012) }\end{array}$ & $\begin{array}{l}\text { Several housing } \\
\text { options (including } \\
\text { special retirement } \\
\text { and congregate } \\
\text { housing) }\end{array}$ & $\begin{array}{l}\text { To examine how sociode- } \\
\text { mographic characteris- } \\
\text { tics, health, and unmet } \\
\text { support needs influ- } \\
\text { ence relocation prefer- } \\
\text { ences }\end{array}$ & Push-pull framework & $\begin{array}{l}\text { Mail-in survey based on the } \\
1998 \text { Seniors' Housing } \\
\text { and Support Services } \\
\text { Survey (developed by } \\
\text { the Canada Mortgage } \\
\text { and Housing Corpor- } \\
\text { ation) } \\
\text { Self-reported health status } \\
\text { (excellent, very good, } \\
\text { good, fair, or poor); Dif- } \\
\text { ficulty in completing at } \\
\text { least } 1 \text { ADL }\end{array}$ & Logistic regression & $\begin{array}{l}1,015 \text { community- } \\
\text { dwelling adults } \\
\text { (58\% female) in } 4 \\
\text { Canadian prov- } \\
\text { inces }\end{array}$ & $\begin{array}{l}34 \% 65-69 \text { years } \\
27 \% 70-74 \text { years } \\
20 \% 75-79 \text { years } \\
11 \% 80-84 \text { years } \\
7 \% \geq 85 \text { years }\end{array}$ \\
\hline $\begin{array}{l}\text { Ewen and Chahal } \\
\text { (2013) }\end{array}$ & $\begin{array}{l}\text { Congregate senior } \\
\text { housing }\end{array}$ & $\begin{array}{l}\text { To elucidate push-pull } \\
\text { factors associated with } \\
\text { moving into congre- } \\
\text { gate senior housing }\end{array}$ & Push-pull framework & $\begin{array}{l}\text { Semi-structured interviews } \\
\text { and scales for measuring } \\
\text { psychosocial well-being } \\
\text { Self-rated health (Cantril } \\
\text { ladder; } 10=\text { =ery best } \\
\text { possible health and } \\
0=\text { worst possible health); } \\
\text { co-morbid health condi- } \\
\text { tions }\end{array}$ & $\begin{array}{l}\text { Horizontalization, } \\
\text { clusters of } \\
\text { meaning, and } \\
\text { developing } \\
\text { themes. } \\
\text { Multiple regression } \\
\text { and } t \text {-tests for } \\
\text { quantitative } \\
\text { data }\end{array}$ & $\begin{array}{l}26 \text { older women who } \\
\text { recently moved to } \\
\text { congregate senior } \\
\text { housing }\end{array}$ & Mean: 78 years \\
\hline $\begin{array}{l}\text { Walker and } \\
\text { McNamara } \\
\text { (2013) }\end{array}$ & $\begin{array}{l}\text { Retirement living facil- } \\
\text { ities }\end{array}$ & $\begin{array}{l}\text { To identify issues older } \\
\text { adults face when } \\
\text { relocating to retirement } \\
\text { living }\end{array}$ & $\begin{array}{l}\text { Person-environment- } \\
\text { occupation model, } \\
\text { Stages of relocation } \\
\text { from decision } \\
\text { (push-pull factors) } \\
\text { to adjustment. }\end{array}$ & Semi-structured interviews & $\begin{array}{l}\text { Grounded theory } \\
\text { approach }\end{array}$ & $\begin{array}{l}16 \text { "relatively healthy" } \\
\text { residents (12 female) } \\
\text { from retirement liv- } \\
\text { ing facilities ( } 3 \text { who } \\
\text { recently made the } \\
\text { decision to relocate) }\end{array}$ & Mean: 80 years \\
\hline
\end{tabular}


Table 3: Continued

\begin{tabular}{|c|c|c|c|c|c|c|c|}
\hline Authors (Year) & $\begin{array}{l}\text { Descriptor for Sup- } \\
\text { portive Housing }\end{array}$ & Purpose of Study & $\begin{array}{l}\text { Guiding Frame- } \\
\text { works or Models }\end{array}$ & $\begin{array}{l}\text { Data Collection Methods } \\
\text { and Tools }\end{array}$ & Data Analysis & $\begin{array}{l}\text { Participants or } \\
\text { Sample }\end{array}$ & $\begin{array}{l}\text { Age Reported } \\
\text { (Years) }\end{array}$ \\
\hline $\begin{array}{l}\text { Crisp, Windsor, } \\
\text { Anstey, and } \\
\text { Butterworth } \\
\text { (2013) }\end{array}$ & Retirement village & $\begin{array}{l}\text { To identify distinguishing } \\
\text { characteristics of older } \\
\text { adults who consider } \\
\text { relocation in the future } \\
\text { from those who have } \\
\text { not }\end{array}$ & $\begin{array}{l}\text { Push-pull framework, } \\
\text { Five factor model of } \\
\text { personality }\end{array}$ & $\begin{array}{l}\text { Mail-in survey } \\
\text { Self-rated physical health in } \\
\text { relation to physical } \\
\text { activity, pain, and gen- } \\
\text { eral perceived health } \\
\text { (RAND-12 Health Status } \\
\text { Inventory [RAND- } \\
\text { 12]) Physical Health } \\
\text { Component Score) }\end{array}$ & Logistic regressions & $\begin{array}{l}517 \text { community- } \\
\text { dwelling residents } \\
\text { (51\% female) }\end{array}$ & $\begin{array}{l}\text { Mean: } 65 \text { years } \\
\text { (SD: 8.0) }\end{array}$ \\
\hline $\begin{array}{l}\text { Crisp, Windsor, } \\
\text { Butterworth, } \\
\text { and Anstey } \\
(2013)\end{array}$ & & $\begin{array}{l}\text { To identify relocation fac- } \\
\text { tors that older adults } \\
\text { find encouraging or } \\
\text { discouraging }\end{array}$ & $\begin{array}{l}\text { Gardner's two types } \\
\text { of movers to } \\
\text { retirement villages: } \\
\text { planners and } \\
\text { reactors. }\end{array}$ & & & & \\
\hline Bohle et al. (2014) & Retirement village & $\begin{array}{l}\text { To explore influences on } \\
\text { the housing choices of } \\
\text { retirees }\end{array}$ & $\mathrm{N} / \mathrm{A}$ & Convergent interviews & $\begin{array}{l}\text { Analysis of con- } \\
\text { vergent and } \\
\text { divergent } \\
\text { themes }\end{array}$ & $\begin{array}{l}81 \text { retirement village } \\
\text { residents ( } 46 \\
\text { female) and } 73 \\
\text { local community } \\
\text { residents ( } 40 \\
\text { female) }\end{array}$ & $\begin{array}{l}\text { Mean: } 77.7 \text { years } \\
\text { (range: } 59-93 \\
\text { years) }\end{array}$ \\
\hline $\begin{array}{l}\text { Granbom, et al. } \\
(2014)\end{array}$ & Special housing & $\begin{array}{l}\text { To explore which aspects } \\
\text { of housing and health } \\
\text { predict relocation to } \\
\text { ordinary or special } \\
\text { housing in very old age }\end{array}$ & $\begin{array}{l}\text { Ecological theory of } \\
\text { ageing (person- } \\
\text { environment fit) }\end{array}$ & $\begin{array}{l}\text { Longitudinal data (part of } \\
\text { the Enabling Autonomy, } \\
\text { Participation, and Well- } \\
\text { Being in Old Age: The } \\
\text { Home Environment as a } \\
\text { Determinant for Healthy } \\
\text { Aging [ENABLE-AGE] } \\
\text { Project) collected via } \\
\text { home visits, interviews, } \\
\text { assessments, and obser- } \\
\text { vations } \\
\text { Symptom List Questionnaire } \\
\text { (0-30); Geriatric Depres- } \\
\text { sion Scale (0-15); } 4 \text { tasks } \\
\text { from } \\
\text { Mini-Mental State Examin- } \\
\text { ation (MMSE) } \\
\text {; use of a mobility device; } \\
\text { ADLs; IADLs; perceived } \\
\text { functional independ- } \\
\text { ence; SF-36 }\end{array}$ & $\begin{array}{l}\text { Cox regression } \\
\text { modeling }\end{array}$ & $\begin{array}{l}384 \text { persons living in } \\
\text { the community } \\
(75 \% \text { female); } 70 \\
\text { participants } \\
\text { relocated after } 4 \\
\text { years ( } 46 \text { to special } \\
\text { housing) }\end{array}$ & $\begin{array}{l}\text { Mean: } 84.9 \text { years } \\
\text { (SD: } 3.0)\end{array}$ \\
\hline
\end{tabular}


Table 3: Continued

\begin{tabular}{|c|c|c|c|c|c|c|c|}
\hline Authors (Year) & $\begin{array}{l}\text { Descriptor for Sup- } \\
\text { portive Housing }\end{array}$ & Purpose of Study & $\begin{array}{l}\text { Guiding Frame- } \\
\text { works or Models }\end{array}$ & $\begin{array}{l}\text { Data Collection Methods } \\
\text { and Tools }\end{array}$ & Data Analysis & $\begin{array}{l}\text { Participants or } \\
\text { Sample }\end{array}$ & $\begin{array}{l}\text { Age Reported } \\
\text { (Years) }\end{array}$ \\
\hline $\begin{array}{l}\text { Rockwood et al. } \\
\text { (2014) }\end{array}$ & $\begin{array}{l}\text { Assisted living and } \\
\text { nursing home }\end{array}$ & $\begin{array}{l}\text { To compare events and } \\
\text { symptoms that predis- } \\
\text { pose and precipitate } \\
\text { moving of older adults } \\
\text { to assisted living or to a } \\
\text { nursing home }\end{array}$ & $\mathrm{N} / \mathrm{A}$ & $\begin{array}{l}\text { Health record review and } \\
\text { personal/facility inter- } \\
\text { view. } \\
\text { Cognitive impairment scale } \\
\text { (Global Deterioration } \\
\text { Scale); Dependence } \\
\text { Scale (0-15, } 15 \text { indicating } \\
\text { worse dependence); } \\
\text { Dementia Symptom } \\
\text { Scale (SymptomGuide) }\end{array}$ & $\begin{array}{l}\text { Analysis of vari- } \\
\text { ance and chi- } \\
\text { square }\end{array}$ & $\begin{array}{l}174 \text { residents who } \\
\text { were admitted to } \\
\text { nursing home or } \\
\text { assisted living; } 54 \\
\text { moving from com- } \\
\text { munity to assisted } \\
\text { living (69\% female) }\end{array}$ & $\begin{array}{l}\text { Mean: } 85.3 \text { years } \\
\text { (SD: } 6.4) \text { mov- } \\
\text { ing to assisted } \\
\text { living }\end{array}$ \\
\hline $\begin{array}{l}\text { Crisp, Windsor, } \\
\text { Butterworth, } \\
\text { and Anstey } \\
(2015)\end{array}$ & Retirement community & $\begin{array}{l}\text { To investigate changes in } \\
\text { loneliness or social net- } \\
\text { works following } \\
\text { relocation }\end{array}$ & $\begin{array}{l}\text { Five Factor Personal- } \\
\text { ity Model } \\
\text { Social network-health } \\
\text { relationship }\end{array}$ & $\begin{array}{l}\text { Mailed-in self-report ques- } \\
\text { tionnaires at prior to } \\
\text { relocation, } 1,6 \text {, and } 12 \\
\text { months post relocation } \\
\text { SF-12 Physical Health, } \\
\text { SF-12 Mental Health; } \\
\text { Depression Anxiety Stress } \\
\text { Scales (DASS) }\end{array}$ & $\begin{array}{l}\text { Longitudinal mod- } \\
\text { eling }\end{array}$ & $\begin{array}{l}83 \text { older adults } \\
\text { relocating to a new } \\
\text { retirement commu- } \\
\text { nity (61\% female); } \\
549 \text { community- } \\
\text { dwelling older } \\
\text { adults }\end{array}$ & $\begin{array}{l}\text { Mean age of } \\
\text { those moving to } \\
\text { retirement } \\
\text { homes: } 75 \\
\text { years (SD: } 7.66 \text { ) }\end{array}$ \\
\hline Ayalon (2016) & CCRC & $\begin{array}{l}\text { To evaluate views of } \\
\text { CCRC residents on the } \\
\text { nursing unit within their } \\
\text { community }\end{array}$ & $\mathrm{N} / \mathrm{A}$ & $\begin{array}{l}\text { Interviews conducted over } \\
4 \text { years } \\
\text { Subjective health status } \\
\text { (excellent, good, medi- } \\
\text { ocre, poor, very poor) }\end{array}$ & $\begin{array}{l}\text { Longitudinal the- } \\
\text { matic analysis }\end{array}$ & $\begin{array}{l}57 \text { continuing care } \\
\text { retirement commu- } \\
\text { nity residents } \\
\text { ( } 60 \% \text { female) }\end{array}$ & $\begin{array}{l}\text { Mean: } 80 \text { years } \\
\text { (SD: } 4.7 \text { ) (first } \\
\text { interview) to } 83 \\
\text { years (SD: } 3.5 \text { ) } \\
\text { (last interview) }\end{array}$ \\
\hline $\begin{array}{l}\text { Portacolone and } \\
\text { Halpern (2016) }\end{array}$ & Housing for seniors & $\begin{array}{l}\text { To understand reasons } \\
\text { that led community- } \\
\text { dwelling older adults to } \\
\text { relocate to senior } \\
\text { housing }\end{array}$ & $\begin{array}{l}\text { Micro/subjective, } \\
\text { meso/institutional, } \\
\text { and macro/ideo- } \\
\text { logical lens of } \\
\text { analysis }\end{array}$ & $\begin{array}{l}\text { Ethnographic interviews } \\
\text { and participant obser- } \\
\text { vation }\end{array}$ & $\begin{array}{l}\text { Thematic analysis } \\
\text { guided by } \\
\text { micro, meso, or } \\
\text { macro lens of } \\
\text { analysis }\end{array}$ & $\begin{array}{l}23 \text { living in building } \\
\text { for seniors and } 24 \\
\text { living in conven- } \\
\text { tional housing ( } 66 \% \\
\text { female); all living } \\
\text { alone }\end{array}$ & $\geq 75$ years \\
\hline $\begin{array}{l}\text { Koss and Ekerdt } \\
\text { (2016) }\end{array}$ & Supportive housing & $\begin{array}{l}\text { To examine how antici- } \\
\text { pation of the fourth } \\
\text { age influences third- } \\
\text { age residential reason- } \\
\text { ing }\end{array}$ & $\begin{array}{l}\text { Ecological theory of } \\
\text { aging } \\
\text { Anticipation of the } \\
\text { fourth age } \\
\text { (marked by pro- } \\
\text { gressive depend- } \\
\text { ence and decline) }\end{array}$ & Semi-structured interviews & Thematic analysis & $\begin{array}{l}30 \text { community dwell- } \\
\text { ing older adults } \\
\text { (50\% female). } 7 \\
\text { lived in independ- } \\
\text { ent housing in or } \\
\text { associated with } \\
\text { age-segregated } \\
\text { communities }\end{array}$ & $\begin{array}{l}\text { Mean: } 76.4 \text { years } \\
\text { (range: } 67-97 \\
\text { years) }\end{array}$ \\
\hline
\end{tabular}


Table 4: Theoretical frameworks

\begin{tabular}{|c|c|c|}
\hline Framework & Brief Description & Key Article \\
\hline Push and pull framework & See Introduction. & Tyvimaa \& Kemp (2011) \\
\hline Ecological theory of aging & $\begin{array}{l}\text { There are age-related changes to health and function that influence the person- } \\
\text { environment fit. This causes an "environmental press", which may result in } \\
\text { relocation. }\end{array}$ & Granbom et al. (2014) \\
\hline $\begin{array}{l}\text { Litwak and Longino's model of three } \\
\text { types of moves among the elderly }\end{array}$ & $\begin{array}{l}\text { Older adults make three types of moves: (1) healthy retirees moving for amenities } \\
\text { and friendship, (2) frail older adults moving because of their need for informal } \\
\text { care, and (3) older adults moving because of their increased dependency and } \\
\text { need for formal care. }\end{array}$ & Krout et al. (2002) \\
\hline Gardner's two types of movers & $\begin{array}{l}\text { There are two types of movers: (1) planners who are motivated by future health } \\
\text { concerns and (2) reactors who move because of current health problems. }\end{array}$ & $\begin{array}{l}\text { Crisp, Windsor, Anstey, \& } \\
\text { Butterworth (2013) }\end{array}$ \\
\hline $\begin{array}{l}\text { Golant's model of an interactional } \\
\text { worldview }\end{array}$ & $\begin{array}{l}\text { Builds on ecological models by providing a temporal perspective on person- } \\
\text { environment and behavioural relationships. }\end{array}$ & Smith and Sylvestre (2008) \\
\hline $\begin{array}{l}\text { Complementary/ congruence model } \\
\text { of wellbeing }\end{array}$ & $\begin{array}{l}\text { Older adults' well-being is dependent on their ability to satisfy their needs using } \\
\text { available environmental resources. }\end{array}$ & Hong and Chen (2009) \\
\hline Therapeutic landscapes & $\begin{array}{l}\text { A concept used by health geographers in which one's outcomes are influenced by } \\
\text { the power of place to provide physical, mental, and spiritual healing. }\end{array}$ & Cheng et al. (2011) \\
\hline Ecological systems & $\begin{array}{l}\text { The use of micro/subjective, meso/institutional, and macro/ideological perspectives } \\
\text { to understand relocation. }\end{array}$ & Portacolone \& Halpern (2016) \\
\hline $\begin{array}{l}\text { Rosenbaum's theory of learned } \\
\text { resourcefulness }\end{array}$ & $\begin{array}{l}\text { Learned resourcefulness (a repertoire of self-control skills and ability to cope with } \\
\text { adversity) can facilitate positive relocations. }\end{array}$ & Bekhet et al. (2008) \\
\hline Anticipatory and disenfranchised grief & $\begin{array}{l}\text { Anticipatory grief refers to one's reaction to impending losses. Disenfranchised grief } \\
\text { refers to grief that one cannot openly acknowledge. }\end{array}$ & Ayalon \& Green (2012) \\
\hline Theory of planned behaviour & $\begin{array}{l}\text { States that one's behaviours are determined by one's intention to perform that } \\
\text { action, and that such intention is influenced by attitude and the subjective norm. }\end{array}$ & Huang (2012) \\
\hline
\end{tabular}

Pull factors for relocation were related to one's lifestyle, community and social amenities, the prospect of receiving care, and affordability (Table 5). Pull factors generally involved the availability of amenities and care that enabled older adults to maintain an existing lifestyle (Stimson \& McCrea, 2004). Articles that did not apply the push and pull framework also suggested that reasons for relocation related to the maintenance of older adults' current lifestyle. For example, Kemp (2008) found that couples who moved to assisted living homes did so because of their desire to continue living together after a spouse's major health transition. The push and pull factors are described in Table 5.

\section{Other Factors Influencing Relocation}

Articles that used other models or no explicit guiding conceptual framework described additional factors, which may or may not be related to push and pull factors, influencing older adults' relocation to supportive housing. An article that used the ecological theory of ageing examined how different dimensions affected relocation: functional status, features of current housing, social networks, features of retirement communities, and finances (Caro et al., 2012; Sergeant \& Ekerdt, 2008). Another article described how increasing dependence results in changes in the person-environment fit, which may precipitate the move (Granbom et al., 2014). Using the ecological theory of ageing, Koss and Ekerdt (2016) categorised older adults' reasoning for relocation as preemptive, where participants believed that their current homes would be suitable in the future, or contingent, where they have anticipated having the need to relocate.

Reviewed articles also explored the impact of adult children (Castle \& Sonon, 2007; Sylvestre \& Smith, 2009), older adults' subjective interpretations of the new residential setting (Smith \& Sylvestre, 2008), socioeconomic status and race (Ball et al., 2009), learned resourcefulness (Bekhet, Zauszniewski, \& Wykle, 2008), grief (Ayalon \& Green, 2012), and the larger cultural and political context (Portacolone \& Halpern, 2016; Sergeant \& Ekerdt, 2008) as factors for relocation.

\section{Study Participants}

All articles reported participants' gender, and all but one $(97 \%)$ reported participants' age (Table 2$)$. With the 
Table 5: Push and pull factors affecting relocation

\begin{tabular}{|c|c|c|}
\hline Study & Push Factors & Pull Factors \\
\hline Groger \& Kinney (2001) & $\begin{array}{l}\text { Desire to plan while able } \\
\text { Optimal timing and ready for change } \\
\text { Fear of burdening family }\end{array}$ & $\begin{array}{l}\text { Attachment to community } \\
\text { Joining friends and neighbors } \\
\text { Proximity to family } \\
\text { Prospect of long-term care }\end{array}$ \\
\hline Krout et al. (2002) & $\begin{array}{l}\text { Decline in own or spouse's health } \\
\text { Deteriorating homes } \\
\text { Reduction in income }\end{array}$ & $\begin{array}{l}\text { Continuing care } \\
\text { Health services on site } \\
\text { Household and maintenance help }\end{array}$ \\
\hline Stimson \& McCrea (2004) & $\begin{array}{l}\text { Change in lifestyle } \\
\text { Home maintenance } \\
\text { Social isolation } \\
\text { Health and mobility }\end{array}$ & $\begin{array}{l}\text { Built environment } \\
\text { Affordability } \\
\text { Location } \\
\text { Desire to maintain existing lifestyle }\end{array}$ \\
\hline Bekhet et al. (2009) & $\begin{array}{l}\text { Own or spouse's failing health } \\
\text { Getting rid of responsibilities } \\
\text { No help } \\
\text { Closing facility } \\
\text { Loneliness }\end{array}$ & $\begin{array}{l}\text { Location } \\
\text { Family and reputation of facility } \\
\text { Security } \\
\text { Joining friends }\end{array}$ \\
\hline Tyvimaa \& Kemp (2011) & $\begin{array}{l}\text { Personal circumstances: social isolation, declining health } \\
\text { status, need for assistance } \\
\text { Physical environment: heavy housework, renovation } \\
\text { Community environment: unsafe neighbourhood, lack } \\
\text { of services }\end{array}$ & $\begin{array}{l}\text { Social environment: new lifestyle, activities, age homo- } \\
\text { geneity } \\
\text { Physical environment: easy living, purpose built } \\
\text { Community environment: location, access to services, } \\
\text { public transportation }\end{array}$ \\
\hline Bäumker et al. (2012) & $\begin{array}{l}\text { Health reasons (e.g. own or spouse's health) } \\
\text { Housing reasons (e.g. home requiring adaptation or too } \\
\text { much to manage) } \\
\text { Social reasons (e.g. isolation) were less important than } \\
\text { health and housing reasons }\end{array}$ & $\begin{array}{l}\text { Attractions of extra care (e.g. tenancy rights, care } \\
\text { support on-site, security, accessibility, size, communal } \\
\text { areas, family/friends, social//eisure facilities) }\end{array}$ \\
\hline Weeks et al. (2012) & $\begin{array}{l}\text { Authors did not categorize factors. Factors significantly } \\
\text { years old), household income (higher income), whethe } \\
\text { cleaning needs. }\end{array}$ & $\begin{array}{l}\text { lated to relocation included gender (women), age }(<80 \\
\text { the current home met their needs, and unmet heavy }\end{array}$ \\
\hline Ewen \& Chahal (2013) & $\begin{array}{l}\text { Health and functional decline } \\
\text { To relocate closer to a relative who needs care }\end{array}$ & Availability of on-site services \\
\hline $\begin{array}{l}\text { Crisp, Windsor, Anstey, \& Butterworth } \\
\text { (2013) }\end{array}$ & \multicolumn{2}{|c|}{$\begin{array}{l}\text { Authors did not categorize factors. Those most likely to have considered relocation to retirement villages were: } \\
\text { younger (55-64 years old vs. } 65-74) \text {, or retirees with enough money, worse physical health, and poorer current } \\
\text { neighbourhood social cohesion. }\end{array}$} \\
\hline
\end{tabular}

exception of one study that included only women (Saunders \& Heliker, 2008), 60-70 per cent of participants were women (Table 3). All mean and median ages were greater than 60 years (Table 3). Younger participants (with a mean age of 65 years old) tended to be community-dwelling residents who may have been relocating to supportive housing (Crisp, Windsor, Anstey, \& Butterworth,, 2013; Weeks, Keefe, \& Macdonald, 2012). In contrast, in articles with participants who were already living in supportive housing, the participants were 70-80 years old (Bäumker et al., 2012).

Participants were often described as healthy (Walker \& McNamara, 2013) and/or cognitively unimpaired (Bekhet et al., 2008); no studies focused on older adults with significant physical and/or cognitive impairments. Approximately two thirds of studies used at least one measure of health or functioning (Table 6). Up to 62 per cent assessed general health, with self-rated health being the most frequently used instrument. Activities of daily living (ADLs) and instrumental activities of daily living (IADLs) were the second most frequently assessed aspect of health and functioning, with 32 per cent of studies applying an instrument to measure them. Other standardized instruments, such as the Center for Epidemiologic Studies Depression Scale (CES-D) and the Minimum Data Set Cognitive Performance Scale (MDSCPS), were used to measure depression / mood and cognition, respectively (Table 6). A total of 12 per cent of studies collected information related to specific health conditions from patients and/or their medical records (Ball et al., 2009; Ewen \& Chahal, 2013; Hong \& Chen, 2009; Sergeant \& Ekerdt, 2008). Cardiovascular disease and hypertension were the most commonly reported 
Table 6: Instruments to assess health and/or function

\begin{tabular}{|c|c|}
\hline $\begin{array}{l}\text { Aspect of Health } \\
\text { or Functioning }\end{array}$ & Instruments \\
\hline General health & $\begin{array}{l}\text { Self-rated health status (usually using } \\
\text { a 5-point Likert scale) } \\
\text { Short Form Survey-36 (SF-36) } \\
\text { Symptom List Questionnaire }\end{array}$ \\
\hline $\begin{array}{l}\text { Activities of daily living (ADLs)/ } \\
\text { instrumental ADLs (IADLs) }\end{array}$ & $\begin{array}{l}\text { Index of Activities of Daily Living } \\
\text { ADL and IADL status } \\
\text { Functional disability (Nagi's } 7 \text { items) } \\
\text { Perceived functional independence }\end{array}$ \\
\hline Depression or mood & $\begin{array}{l}\text { Center for Epidemiologic Studies } \\
\text { Depression Scale (CES-D) } \\
\text { Mood scale (0-30) } \\
\text { Geriatric Depression Scale } \\
\text { Depression Anxiety Stress Scale } \\
\text { (DASS) }\end{array}$ \\
\hline Cognition & $\begin{array}{l}\text { Metamemory Questionnaire } \\
\text { Minimum Data Set Cognitive Per- } \\
\text { formance Scale (MDS-CPS) } \\
\text { Four tasks from the Mini Mental State } \\
\text { Examination (MMSE) } \\
\text { Global Deterioration Scale }\end{array}$ \\
\hline Pain & Pain 6-point Likert scale \\
\hline
\end{tabular}

health conditions (Ball et al., 2009; Ewen \& Chahal, 2013; Sergeant \& Ekerdt, 2008).

Articles that commented on participants' health or functioning generally stated that participants were in good physical health with only minor problems. For example, Groger and Kinney (2001) reported that participants had high levels of well-being, with the exception of a few reporting minor "forgetfulness" problems. Studies that used self-rated health as a measure of general health commonly reported that participants were in "fair" or "excellent" health (Bekhet et al., 2009; Huang, 2012; Weeks et al., 2012). Some of the articles suggest some deficits in ADLs/IADLs among study participants. One study reported average scores of 7.2/10 and 5.5/10 on the Older Americans Resources and Services ADL and IADL Scales (Castle \& Sonon, 2007), whereas another found that only 31 per cent of 215 retirement home residents were independent with two or more IADLs (Caro et al., 2012). The three articles that examined how health and functioning impacted relocation found that worse health, dependence with IADLs, cognitive deficits, and accessibility problems were associated with moving to supportive housing (Granbom et al., 2014; Hong \& Chen, 2009; Rockwood et al., 2014).

\section{Discussion}

We conducted a scoping review to identify and describe manuscripts reporting on older adults' reasons for relocation to supportive housing. Of the 34 articles that met eligibility criteria, 12 per cent described studies that were conducted in Canada; the majority were published after 2007. Thirty-five percent of articles were published in a journal not indexed for MEDLINE, which may hinder their visibility to health services researchers. As a result, literature regarding older adults' reasons for relocating to supportive housing may be under-utilised to inform the planning and delivery of care, and refinement of supportive policy. This may also explain why the literature may focus on the geographical and planning aspects of older adults' relocation rather than health-related factors.

Articles reviewed were heterogeneous. First, numerous descriptors were used to designate purpose-built housing that provides services for older adults, ranging from "senior housing" to "retirement homes". This is consistent with previous reviews of supportive housing nomenclature, suggesting that commonalities exist among settings despite the diversity in descriptors used (Howe et al., 2013). Second, studies employed a variety of qualitative and quantitative designs. Despite differing approaches, both qualitative and quantitative studies had a shared purpose: to understand the factors driving older adults' relocation to supportive housing. Notably, some articles reported using similar frameworks despite using different study designs. For example, Groger and Kinney (2001) used the push and pull framework to analyze interview data, whereas Stimson and McCrea (2004) used the framework to guide the development of a model from survey data.

The use of a guiding framework or model was reported in 76 per cent of manuscripts. One third of articles that used a guiding model explicitly applied the push and pull framework, making it the most frequently used conceptual framework. Another commonly used conceptual framework was the ecological theory of ageing, which revolves around the person-environmental fit (Granbom et al., 2014). Despite the use of different guiding frameworks and models, there appears to be a common theme among the reviewed articles: a combination of push and pull factors influences older adults' relocation to supportive housing. For example, "environmental press", as described in the ecological theory of ageing, is analogous to push factors. Another example includes the Gardner's model of two types of movers that categorises older adults into planners and reactors (Crisp, Windsor, Butterworth, \& Anstey, 2013), echoing that some are pushed into relocating to supportive housing and must move reactively, whereas others may be pulled into relocating by planning around their anticipated future needs.

Generally, the reviewed studies, specifically those using qualitative approaches, provide valuable insight into 
the influence of older adults' lived experiences, albeit framed a priori using guiding models, on their relocation to supportive housing. Perceived and actual decline in health or health of a spouse were the most commonly cited push factors. Pull factors generally revolved around the availability of amenities and support that participants anticipated that they would need in the future. Importantly, these factors are also consistent with the results of articles which did not explicitly utilise the push and pull framework, suggesting that these findings are not just artifacts resulting from the use of this guiding model. Articles also explored potentially influential variables, such as the role of adult children and grief, which modify older adults' experiences with relocation but do not necessarily push or pull them towards supportive housing.

Overall, studies that included both community-dwelling and supportive housing residents showed that those residing in supportive housing tended to be older and were mostly women (Crisp, Windsor, Anstey, \& Butterworh, 2013; Weeks et al., 2012). This may be because women have a longer life expectancy than men, and because of the association between increasing age and health and functional deficits. The likelihood that women are the surviving partner in their relationship may contribute to their relative overrepresentation in supportive housing. Many men with similar health and functional challenges may have partners to help them avoid moving to supportive housing (Rockwood, Song, \& Mitnitski, 2011). Approximately two thirds of articles used at least one measure of health or function, and most participants were described as healthy, with a few being described as having minor deficits in functioning. However, three articles examined the impact of health and functioning on relocation to supportive housing (Granbom et al., 2014; Hong \& Chen, 2009; Rockwood et al., 2014). These manuscripts reported that physical impairments and functional impairments were associated with moving to supportive housing. The instruments used to assess health and function varied and often relied on self-report. The limited and largely subjective data on participants' health and functioning hinder the extrapolation of whether needs are met in supportive housing.

This review of 34 articles reporting on factors surrounding older adults' relocation to supportive housing revealed several gaps in the literature. First, the results of reviewed articles suggest that older adults are pushed into supportive housing by declining physical health and functioning. However, details about this decline, such as diagnoses and comorbidities, are limited by the variable use of instruments and reliance on self-report. Second, there is a collage of different terms used to describe supportive housing, which hinders comparisons and policy discussions with regard to this setting (Howe et al., 2013). Third, financial considerations were identified in a small number of studies, which is surprising given the costs often associated with supportive housing options (Federal/Provincial/Territorial Ministers Responsible for Seniors, 2019). Moreover, considerations related to gender identity, culture, and religion appear to be virtually absent from the literature. Lastly, evidence regarding supportive housing consists of both health-related and non-health-related literature. Although this body of evidence facilitates a multidimensional understanding of older adults' relocation to supportive housing, active efforts may be required to bridge silos between disciplines.

Gaps identified in this review make it difficult to ascertain the appropriateness of current policies. Although evidence suggests that older adults relocate to supportive housing in part because of health and functional impairments, there appears to be a paucity of comprehensive and observational literature to support this. In Canada, the Federal/ Provincial/Territorial Ministers Responsible for Seniors (2019) recently called for more evidence that considers the many factors at play, including socio-economic and cultural ones, to guide policies for older adults' housing. Future research should focus on collecting and summarising objective information about the health and functioning of older adults relocating to supportive housing. Longitudinal observational study designs may be particularly useful because the current literature suggests that changes in older adults' health and functioning often prompt relocation. This study design can facilitate a detailed understanding of older adults' needs, and consequently, inform policies relevant for both older adults contemplating moving to and those already residing in supportive housing. The application of guiding frameworks and models appears to be useful in exploring health-related and non-health-related factors that influence the transition to supportive housing. However, the use of a framework such as Andersen's behavioral model of health services use (Babitsch, Gohl, \& von Lengerke, 2012) may be more comprehensive in capturing predisposing, enabling, and need factors associated with relocation.

Finally, standardized nomenclature for supportive housing needs to be established to facilitate the synthesis of this evidence, and national and international comparisons of related policies. The mandatory use of interRAI standardized assessments systems in the long-term care and home care sectors across Canada provides a rich resource with which to better understand the clients served in these sectors and guide policy (Heckman, Gray, \& Hirdes, 2013). It is time for a similar approach to be implemented in the supportive housing sector.

\section{Strengths and Limitations}

Our scoping review should be interpreted in light of its strengths and limitations. The strengths of this review 
are the non-restrictive inclusion criteria that encompassed all study types, the use of multiple databases spanning multiple disciplines, and the use of a systematic process documented using reference management software. This review is limited by the exclusion of nonEnglish articles. Finally, our focus was on the identification of factors related to relocation decisions. A number of articles identified also addressed lived experience of the actual relocation and of its aftermath on quality of life in a supportive care setting, which, as important topics, would require specific reviews and further research.

\section{Conclusion}

This scoping review describes the nature and content of 34 articles focusing on older adults' reasons for relocating to supportive housing. Approximately one third of included articles were published in journals not indexed for MEDLINE, which suggests that a portion of literature focuses on non-health-related aspects of supportive housing, such as geography and planning. This is also reflected in the heterogeneous study characteristics that included various qualitative and quantitative designs and different guiding conceptual theories. Ideas explicitly or implicitly related to the push and pull framework were common in the articles. It was frequently reported that declining health and functioning was a commonly cited push factor towards relocation to supportive housing. However, although two thirds of the articles utilised a measure of health or functioning, most relied on subjective and self-reported measures. Future research is needed to produce data regarding the health and functioning of older adults moving to supportive housing to better inform policies for this growing population.

\section{References}

Ajzen, I. (1985). From intentions to actions: A theory of planned behavior. In J. Kuhl \& J. Beckman (Eds.), Action-control: From cognition to behavior (pp. 11-39). Heidelberg, Germany: Springer.

Arksey, H., \& O’Malley, L. (2005). Scoping studies: Towards a methodological framework. International Journal of Social Research Methodology, 8(1), 19-32. https://doi.org/10. $1080 / 1364557032000119616$

Armstrong, R., Hall, B. J., Doyle, J., \& Waters, E. (2011). 'Scoping the scope' of a Cochrane review. Journal of Public Health, 33(1), 147-150. https://doi.org/10.1093/ pubmed/fdr015.

Ayalon, L. (2016). Do not hear, see, or speak: views of older residents and their adult children about the nursing unit in the continuing care retirement community. International Psychogeriatriatrics, 28(11), 1867-1877. doi: $10.1017 /$ s1041610216000788.
Ayalon, L., \& Green, V. (2012). Grief in the initial adjustment process to the continuing care retirement community. Journal of Aging Studies, 26(4), 394-400. doi:10.1016/j. jaging.2012.05.001.

Babitsch, B., Gohl, D., \& von Lengerke, T. (2012). Re-revisiting Andersen's behavioral model of health services use: A systematic review of studies from 1998-2011. Psychosocial Medicine, 9, 1-15. https:/ /doi.org/10.3205/psm000089.

Ball, M. M., Perkins, M. M., Hollingsworth, C., Whittington, F. J., \& King, S. V. (2009). Pathways to assisted living: The influence of race and class. Journal of Applied Gerontology, 28, 81-108. https://doi.org/10.1177/ 0733464808323451.

Bäumker, T., Callaghan, L., Darton, R., Holder, J., Netten, A., \& Towers, A. M. (2012). Deciding to move into extra care housing: Residents' views. Ageing $\mathcal{E}$ Society, 32(7), 1215-1245. https:/ / doi.org/10.1017/S0144686X11000869.

Bekhet, A., Zauszniewski, J. A., \& Nakhla, W. E. (2009). Reasons for relocation to retirement communities: A qualitative study. Western Journal of Nursing Research, 31 (4), 462-479. https: / / doi.org/10.1177/0193945909332009.

Bekhet, A. K., Zauszniewski, J. A., \& Wykle, M. L. (2008). Milieu change and relocation adjustment in elders. Western Journal of Nursing Research, 30(1), 113-129. https:/ /doi.org/10.1177/0193945907309309

Bohle, P., Rawlings-Way, O., Finn, J., Ang, J., \& Kennedy, D. J. (2014). Housing choice in retirement: community versus separation. Housing Studies, 29(1), 108-127. doi:10. 1080/02673037.2013.825693.

Canadian Centre for Elder Law. (2008). Discussion paper on assisted living: Past, present and future legal trends. Retrieved 22 June 2019 from https://www.bcli.org/ publication/discussion-paper-assisted-living-pastpresent-and-future-legal-trends-canada-0

Caro, F. G., Yee, C., Levien, S., Gottlieb, A. S., Winter, J., McFadden, D. L., et al. (2012). Choosing among residential options: Results of a vignette experiment. Research on Aging, 34(1), 3-33. https:/ / doi.org/10.1177/ 0164027511404032.

Carpenter, I., \& Hirdes, J. P. (2013). Using interRAI assessment systems to measure and maintain quality of long-term care A good life in old age? Monitoring and improving quality long-term care (pp. 93-139). Paris: OECD Publishing.

Castle, N. G., \& Sonon, K. E. (2007). The search and selection of assisted living facilities by elders and family. Medical Care, 45(8), 729-738. https://doi.org/10.1097/ MLR.0b013e3180618b6e.

Cheng, Y., Rosenberg, M. W., Wang, W., Yang, L., \& Li, H. (2011). Aging, health and place in residential care facilities in Beijing, China. Social Science \& Medicine, 72 (3), 365-372.

Crisp, D. A., Windsor, T. D., Anstey, K. J., \& Butterworth, P. (2013). Considering relocation to a retirement village: Predictors from a community sample. Australasian 
Journal on Ageing, 32(2), 97-102. https://doi.org/ 10.1111/j.1741-6612.2012.00618.x.

Crisp, D. A., Windsor, T. D., Butterworth, P., \& Anstey, K. J. (2013). What are older adults seeking? Factors encouraging or discouraging retirement village living. Australasian Journal on Ageing, 32(3), 163-170. https:// doi.org/10.1111/j.1741-6612.2012.00623.x.

Crisp, D. A., Windsor, T. D., Butterworth, P., \& Anstey, K. J. (2015). Adapting to retirement community life: Changes in social networks and perceived loneliness. Journal of Relationships Research, 6, e9. https://doi.org/10.1017/ jrr.2015.5.

Ewen, H. H., \& Chahal, J. (2013). Influence of late life stressors on the decisions of older women to relocate into congregate senior housing. Journal of Housing for the Elderly, 27(4), 392-408. https://doi.org/10.1080/ 02763893.2013 .813428 .

Federal/Provincial/Territorial Ministers Responsible for Seniors. (2019). Report on housing needs of seniors. Retrieved 22 June 2019 from: https: / / www.canada.ca / en/employment-social-development/corporate/ seniors/forum/report-seniors-housing-needs.html

Finn, J., Mukhtar, V. Y., Kennedy, D. J., Kendig, H., Bohle, P., \& Rawlings-Way, O. (2011). Financial planning for retirement village living: a qualitative exploration. Journal of Housing for the Elderly, 25(2), 217-242. doi:10. 1080/02763893.2011.571107.

Gardner, I. L., Browning, C., \& Kendig, H. (2005). Accommodation options in later life: Retirement village or community living? Australasian Journal on Ageing, 24(4), 188-195. https:/ /doi.org/10.1111/j.1741-6612.2005.00121.

Granbom, M., Lofqvist, C., Horstmann, V., Haak, M., \& Iwarsson, S. (2014). Relocation to ordinary or special housing in very old age: Aspects of housing and health. European Journal of Ageing, 11(1), 55-65. https:/ / doi.org/ $10.1007 /$ s10433-013-0287-3.

Groger, L., \& Kinney, J. M. (2001). Reason for moving to a continuing care retirement community (CCRC). Gerontologist, 41, 138-139.

Heckman, G., Gray, L. C., \& Hirdes, J. (2013). Addressing health care needs for frail seniors in Canada: The role of interRAI instruments. The Canadian Geriatrics Society Journal of Continuing Medical Education, 3(1), 8-16.

Hirdes, J. P., Mitchell, L., Maxwell, C. J., \& White, N. (2011). Beyond the 'iron lungs of gerontology': Using evidence to shape the future of nursing homes in Canada. Canadian Journal on Aging-Revue Canadienne Du Vieillissement, 30(3), 371-390. https://doi.org/ $10.1017 /$ S0714980811000304.

Hong, S., \& Chen, L. (2009). Contribution of residential relocation and lifestyle to the structure of health trajectories. Journal of Aging \& Health, 21(2), 244-265. https: / / doi.org/10.1177/0898264308328960.
Howe, A. L., Jones, A. E., \& Tilse, C. (2013). What's in a name? Similarities and differences in international terms and meanings for older peoples' housing with services. Ageing E Society, 33, 547-578. https: / / doi.org/10.1017/ S0144686x12000086.

Huang, H. C. (2012). Factors influencing intention to move into senior housing. Journal of Applied Gerontology, 31(4), 488-509. https: / / doi.org/10.1177/0733464810392225.

Jungers, C. M. (2010). Leaving home: An examination of latelife relocation among older adults. Journal of Counseling and Development, 88(4), 416-423.

Katz, S., Downs, T. D., Cash, H. R., \& Grotz, R. C. (1970). Progress in development of the index of ADL. The Gerontologist, 10(1_Part_1), 20-30.

Kemp, C. L. (2008). Negotiating transitions in later life: Married couples in assisted living. Journal of Applied Gerontology, 27 (3), 231-251. https: / / doi.org/10.1177/0733464807311656.

Koss, C., \& Ekerdt, D. J. (2016). Residential reasoning and the tug of the fourth age. Gerontologist, 57(5), 921-929. https: / / doi.org/10.1093/geront/gnw010.

Krout, J. A., Moen, P., Holmes, H. H., Oggins, J., \& Bowen, N. (2002). Reasons for relocation to a continuing care retirement community. Journal of Applied Gerontology, 21 (2), 236-256. doi:10.1177/07364802021002007.

Lawton, M. P., \& Brody, E. M. (1970). Assessment of older people: self-maintaining and instrumental activities of daily living. Nursing Research, 19(3), 278.

Lee, E. (1966). A theory of migration. Demography, 3(1), 47-57. https:/ / doi.org/10.2307/2060063.

Levac, D., Colquhoun, H., \& O'Brien, K. K. (2010). Scoping studies: Advancing the methodology. Implementation Science, 5(1), 69. https: / / doi.org/10.1186/1748-5908-5-69.

Litwak, E., \& Longino, C. F., Jr. (1987). Migration patterns among the elderly: A developmental perspective. The Gerontologist, 27(3), 266-272. https:/ / doi.org/10.1093/geront/27.3.266.

Matsoukas, K. (2015). Confirming that a journal is indexed in Medline and/or PubMed. MSK Library Blog. Retrieved 22 June 2019 from https://library.mskcc.org/blog/ 2015/12/ confirming-that-a-journal-is-indexed-inmedline-andor-pubmed/

Ontario Retirement Communities Association. (2018). Types of care. Retrieved 22 June 2019 from http://www.orcareti rement.com/retirement-living/types-of-care/

Perks, T., \& Haan, M. (2010). The dwelling-type choices of older Canadians and future housing demand: An investigation using the Aging and Social Support Survey (GSS16). Canadian Journal on Aging-Revue Canadienne Du Vieillissement, 29(3), 445-463. https:// doi.org/10.1017/S0714980810000413.

Portacolone, E., \& Halpern, J. (2016). "Move or Suffer": Is agesegregation the new norm for older Americans living 
alone. Journal of Applied Gerontology, 35(8), 836-856. https: / / doi.org/10.1177/0733464814538118.

Poss, J. W., Sinn, C. J., Grinchenko, G., Blums, J., Peirce, T., \& Hirdes, J. (2017). Location, location, location: Characteristics and services of long-stay home care recipients in retirement homes compared to others in private homes and long-term care homes. Healthcare Policy, 12(3), 80-93.

Rockwood, J., Richard, M., Garden, K., Hominick, K., Mitnitski, A., \& Rockwood, K. (2014). Precipitating and predisposing events and symptoms for admission to assisted living or nursing home care. Canadian Geriatrics Journal, 17(1), 16-21. https://doi.org/ 10.5770/cgj.17.93.

Rockwood, K., Song, X., \& Mitnitski, A. (2011). Changes in relative fitness and frailty across the adult lifespan: Evidence from the Canadian National Population Health Survey. Canadian Medical Association Journal, 183 (8), E487-E494. https:/ / doi.org/10.1503/cmaj.101271.

Rosenbaum, M. (1990). Introduction: From helplessness to resourcefulness. In $\mathrm{M}$. Rosenbaum (Ed.), Learned resourcefulness, (pp. xxv-xxxv). New York: Springer.

Saunders, J. C., \& Heliker, D. (2008). Lessons learned from 5 women as they transition into assisted living. Geriatric Nursing, 29(6), 369-375. https://doi.org/10.1016/ j.gerinurse.2007.10.018.

Sergeant, J. F., \& Ekerdt, D. J. (2008). Motives for residential mobility in later life: Post-move perspectives of elders and family members. The International Journal of Aging and Human Development, 66(2), 131-154.

Smith, G. C., \& Sylvestre, G. M. (2008). Effects of neighborhood and individual change on the personal outcomes of recent movers to low-income senior housing. Research on Aging, 30(5), 592-617. https:// doi.org/10.1177/0164027508319655.
Statistics Canada. (2017a). Dwellings in Canada. Retrieved 22 June 2019 from http:/ / www12.statcan.gc.ca/censusrecensement/2016/as-sa/98-200-x/2016005/98-200x2016005-eng.cfm

Statistics Canada. (2017b). A portrait of the population aged 85 and older in 2016 in Canada. Retrieved from http:/ / www12.statcan.gc.ca/census-recensement/2016/as-sa/ 98-200-x/2016004/98-200-x2016004-eng.cfm

Stimson, R. J., \& McCrea, R. (2004). A push-pull framework for modelling the relocation of retirees to a retirement village: The Australian experience. Environment and Planning A, 36(8), 1451-1470. https://doi.org/10.1068/ a36206.

Svidén, G., Wikström, B., \& Hjortsjö-Norberg, M. (2002). Elderly persons' reflections on relocating to living at sheltered housing. Scandinavian Journal of Occupational Therapy, 9(1), 10-16. https://doi.org/10.1080/ 110381202753505818

Sylvestre, G. M., \& Smith, G. C. (2009). Spatial aspects of the residential adjustments of older parents moving to lowincome senior housing: A longitudinal study. Geoforum, 40(5), 918-929. https:/ / doi.org/10.1016/j.geoforum.2009.06.007.

Tyvimaa, T., \& Kemp, C. L. (2011). Finnish seniors' move to a senior house: Examining the push and pull factors. Journal of Housing for the Elderly, 25(1), 50-71. https:// doi.org/10.1080/02763893.2011.545742.

Walker, E., \& McNamara, B. (2013). Relocating to retirement living: An occupational perspective on successful transitions. Australian Occupational Therapy Journal, 60 (6), 445-453. https:/ / doi.org/10.1111/1440-1630.12038.

Weeks, L. E., Keefe, J., \& Macdonald, D. J. (2012). Factors predicting relocation among older adults. Journal of Housing for the Elderly, 26(4), 355-371. https:/ / doi.org/ 10.1080/02763893.2011.653099. 